\title{
Optimized Tomographic Acquisition and Reconstruction for Highly Faceted Nanostructures with Isotropic Resolution
}

\author{
P. Ercius* \\ * National Center for Electron Microscopy, Lawrence Berkeley National Laboratory, Berkeley, CA \\ 94720
}

Electron tomography is now an established technique in materials science to recover the threedimensional shape of structures from a series of projections using HAADF-STEM imaging. It is generally believed that the technique is limited to $1 \mathrm{~nm}^{3}$ spatial resolution, insufficient to study faceted nanoparticles $<10 \mathrm{~nm}$ in diameter, but this can be substantially improved if the structure can withstand a large dose and the limitations of the tomography technique are avoided. As the smallest dimensions of nanostructures - such as faceted catalytic nanoparticles - become increasingly important, it is necessary to design tomographic data acquisition and reconstruction techniques to minimize artifacts caused by these limitations. Facets, interfaces and surfaces must be reconstructed with high accuracy to determine the exact three-dimensional shape at high resolution to predict nanoparticle functionality. I will discuss a new tomographic reconstruction algorithm that can utilize images from any projection direction to recover a three-dimensional structure with isotropic resolution. Thus, the high-precision tilt-rotate stage of the TEAM 1 microscope can be utilized to design a tilt-series acquisition to include all information about facets and interfaces. This method can also be used to avoid low-index crystal orientations to eliminate non-linear projected intensities produced by residual diffraction contrast present in HAADF-STEM images.

Faceted nanoparticles are especially susceptible to anisotropic sampling in tomography as their function is directly linked to their exact shape which depends on highly direction dependent information. A single-axis tilt-series can fail to sample important information about a faceted nanoparticle, and the orientation of the particle with respect to the zero-degree projection can influence the reconstructed shape. [1] In Fig. 1a), a simulated reconstruction of a cubic nanoparticle with faces oriented perpendicular to the beam shows the effective loss of cubic faces along the yand z-axes for a single-axis tilt-series. In fig. $1 \mathrm{~b}$ ), a second simulated reconstruction of a single-axis tilt-series shows the same particle rotated $45^{\circ}$ so the [110] edge is normal to the beam. The cubic shape of the particle is now recovered due to better sampling of the shape.

Limitations such as the (infamous) missing wedge and discrete data sampling of the continuous object function produces reconstructions with an-isotropic resolution that can obscure features aligned along certain un- or under-sampled directions. As in Fig 1., this is especially undesirable for faceted nanostructures, because symmetries of an object can be distorted or lost. Fig. 2 shows a representation of the sampling of an object's three-dimensional Fourier transform for a) single-axis and b) dual-axis tilt-series. In a) it is apparent that the missing wedge extends in both the y- and zaxes giving rise to reduced resolution along those directions. In b) the missing wedge is reduced to a missing cone along the original projection direction (z-axis), but the sampling is relatively time/dose inefficient. Alternatively, Fig. 2c) represents the sampling of Fourier space using a continuous tiltrotate scheme with a tilt-rotate stage. The tilt-series contains projections from $\alpha=0-70^{\circ}$ and $\gamma=0-360^{\circ}$ at $5^{\circ}$ and $45^{\circ}$ tilt steps, respectively. Such a tilt-series could conceivably contain projections from any orientation of the object to ensure isotropic resolution. 
Crystalline nanoparticles can also produce diffraction contrast in HAADF-STEM images for tiltangles that unfortunately come close to low-index crystal poles. The resulting erroneous image intensities appear as noise over the expected projected mass-thickness of the object. Normally, this noise is insignificant compared to the averaged intensities of all other projections, but has important consequences for tomography of $<10 \mathrm{~nm}$ structures. Reconstruction near or at atomic resolution requires that the SNR at each projection is sufficient for single atom counting. [2, 3] If the orientation of a single crystalline nanoparticle is known then a continuous tilt-series acquisition can be designed to avoid low-index poles while acquiring information from off-axis directions with equivalent information. [3]

\section{References}

[1] P. Ercius, Microsc. Microanal. 6 (2010) 1192.

[2] J.R. Jinshek, Ultramicrosc. 108 (2008) 589.

[3] S. Van Ert, Nature, 470 (2011) 374.

[3] NCEM funding provided by U.S. Department of Energy DE-AC02-05CH11231

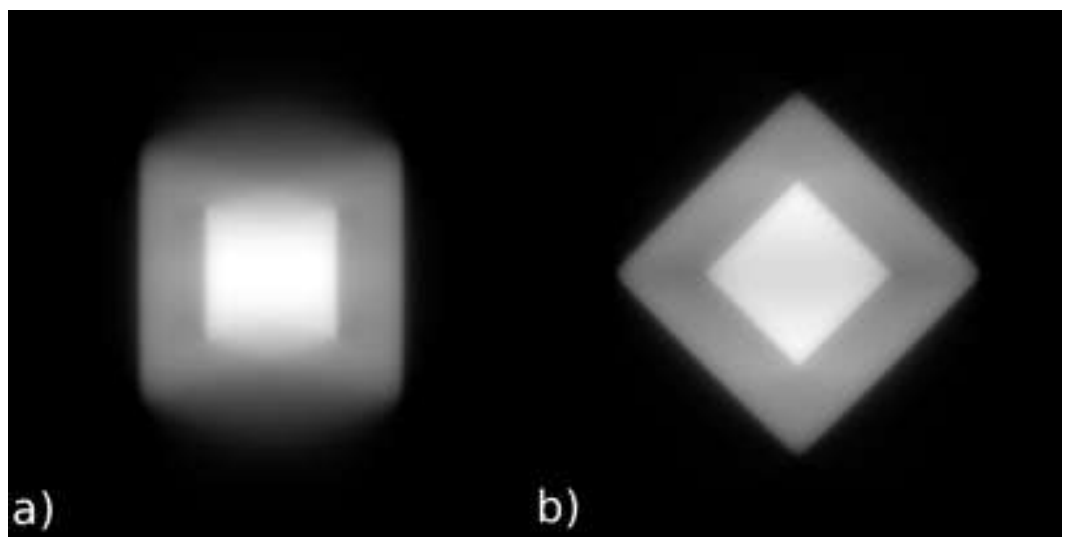

Figure 1. Reconstructions from a single-axis tilt-series for a core-shell, cubic nanoparticle oriented a) perpendicular and b) $45^{\circ}$ with repect to the zero-degree projection direction. The tilt-series fails to sample critical information about the faceted particle in a) but faithfully reconstructs the particle in b). Proper sampling for faceted structures must be considered carefully for true three-dimensional reconstructions.

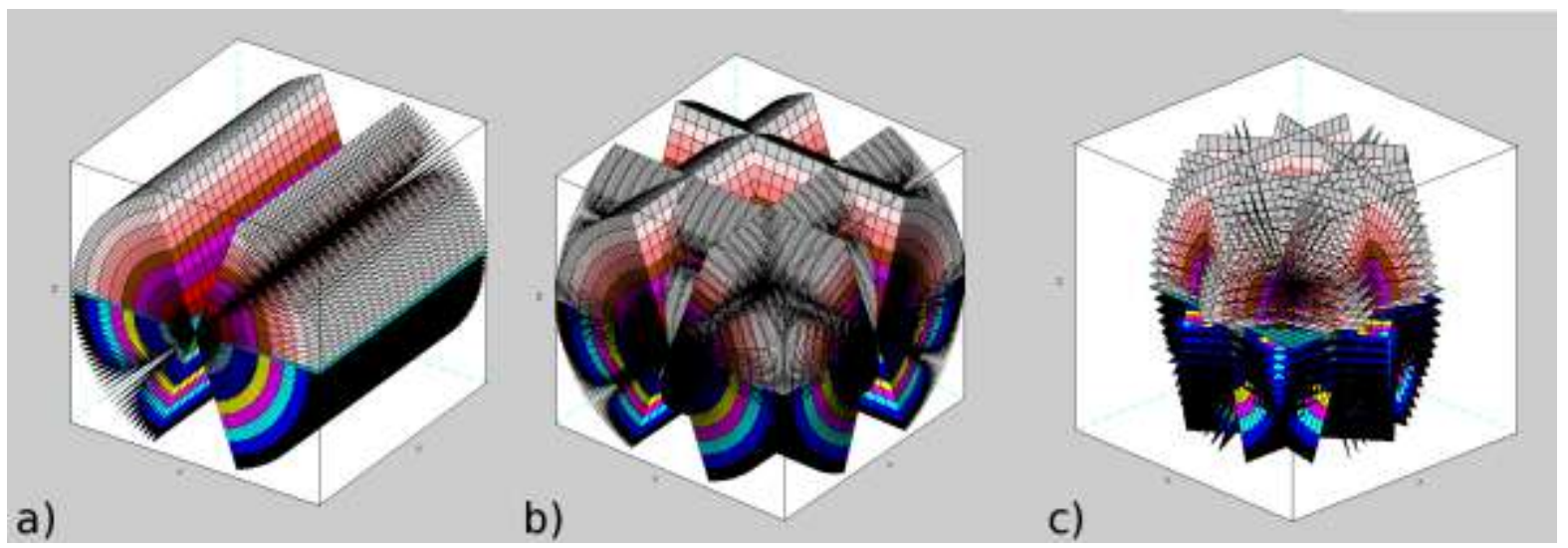

Figure 2. Representation of sampling in Fourier space by a) single-axis $\left(\alpha= \pm 70^{\circ}\right)$, b) dual-axis $\left(\alpha= \pm 70^{\circ}, \gamma=+90^{\circ}\right.$ and $\left.-90^{\circ}\right)$ and $\left.c\right)$ continuous $\left(\alpha=0-70^{\circ}, \gamma=0-360^{\circ}\right)$ tilt-series acquisitions. The alpha axis is parallel to the $X$-axis and the gamma axis is parallel to the $\mathrm{Z}$-axis as in a tilt-rotate stage. The continuous tilt-series efficiently samples information about the object, and produces a reconstruction with isotropic resolution in the $X-Y$ planes and a smaller missing wedge of information compared to a) and b). 